hep-th/0502072

DESY-05-024

\title{
On the one-loop Kähler potential in five-dimensional brane-world supergravity
}

\author{
Adam Falkowski \\ Institute of Theoretical Physics, Warsaw University \\ ul. Hoża 69, PL-00-681 Warsaw, Poland \\ Deutsches Elektronen-Synchrotron DESY \\ Notkestrasse 85, 22607 Hamburg, Germany \\ E-mail: afalkows@mail.desy.de
}

\begin{abstract}
We present an on-shell formulation of $5 \mathrm{~d}$ gauged supergravity coupled to chiral matter multiplets localized at the orbifold fixed points. The brane action is constructed via the Noether method. In such set-up we compute one-loop corrections to the Kähler potential of the effective 4d supergravity and compare the result with previous computations based on the off-shell formalism. The results agree at lowest order in brane sources, however at higher order there are differences. We explain this discrepancy by an ambiguity in resolving singularities associated with the presence of infinitely thin branes.
\end{abstract}




\section{Introduction}

Supersymmetry breaking and its mediation to the observable sector is one of the most important problems in physics beyond the Standard Model. An acceptable theory of supersymmetry breaking is strongly constrained by the observed features of the low-energy physics. Spontaneous breaking must occur in a hidden sector and must be transmitted to the observable sector via non-renormalizable operators. Gravity mediation is an attractive and economical possibility, but it is well known that generic models face the flavor problem.

It has been noted in ref. [1] that a spatial separation of the hidden and observable sectors brings new elements into the mechanism of gravity mediation. The simplest set-up that provides for such sequestering is that of fivedimensional (5d) supergravity compactified on an orbifold in which the chiral matter of the observable and hidden sectors is localized on the two different boundaries of the fifth dimension. In the minimal set-up with no matter fields in the bulk the tree-level Kähler potential of the effective $4 \mathrm{~d}$ theory has a special structure that results in absence of tree-level transmission of supersymmetry breaking. Supersymmetry breaking is then transmitted to the observable sector at one-loop level by (flavor-blind) gravitational interactions. One mechanism that can operate here is anomaly mediation [1, 2]. Besides, there is always a contribution from integrating out the Kaluza-Klein (KK) tower of the supergravity multiplet. Its effect on the low-energy phenomenology can be summarized as a correction to the tree-level Kähler potential of the $4 \mathrm{~d}$ effective supergravity. This one-loop correction was computed in refs. 3, 4, 5, 6. Unlike in four-dimensions, the contact terms between the hidden and the observable sectors generated by gravity loops are finite and calculable. Therefore $5 \mathrm{~d}$ supergravity models open a possibility of constructing a realistic and predictive theory of soft terms. See also [7] for other studies of 5 d brane-world supergravity.

Given the important role of gravitational loop corrections it is advantageous to study them in a somewhat different setting. The brane-world action considered in refs. [3, 4, 5, 6] was based on an off-shell formulation of 5d supergravity. In this paper we point out that the physics of $5 \mathrm{~d}$ brane-worlds can be studied in a simpler set-up of on-shell supergravity. Using the Noether procedure, we construct a locally supersymmetric action for an $N=1$ chiral multiplet confined to a $4 \mathrm{~d}$ brane and coupled to $5 \mathrm{~d}$ gauged supergravity in the bulk. In such set-up we compute the one-loop corrections to the Kähler potential and compare it with the previous results.

In principle, the Noether method is less powerful than the off-shell formalism of ref. [8] or the superconformal tensor calculus of ref. [9]. Still, 
we will argue that it has several advantages. Firstly, it is obviously less involved. The number of fields is reduced and no advanced superspace techniques are needed for constructing the action. We will also see that one-loop computations are considerably simplified in this set-up. Secondly, it facilitates the treatment of singularities associated with the presence of infinitely thin (delta-like) branes. In the off-shell formulation integrating out auxiliary fields generates singular terms in the brane action. These singular terms can be avoided in our Noether formulation. Furthermore, working in our set-up we will notice certain ambiguity in defining the brane-world action that is connected to arbitrariness in resolving the singular behaviour of bulk fields near the branes. In certain circumstances, namely when brane sources are large compared to the compactification scale, this ambiguity may also affect low-energy observables. Finally, the procedure can be readily generalized to higher-dimensional spacetimes where an off-shell formulation of supergravity does not exist (for example, similar method was used for coupling 10d brane to 11d supergravity in the Horava-Witten model [10]).

The paper is organized as follows. In Section 2 we construct an on-shell action for an $N=1$ chiral multiplet coupled to $5 \mathrm{~d}$ supergravity. In Section [3] we derive the tree-level Kähler potential describing the dynamics of the low energy degrees of freedom in this set-up. In Section 4 we compute the one-loop correction to the Kähler potential and in Section [5] we comment on the differences with the previous works. In this paper we restrict to studying technical issues associated with the Noether construction and one-loop computation. Phenomenological issues, like moduli stabilization or determination of soft breaking terms, are left for future publications.

\section{Five-dimensional brane-world action}

In this section we construct a locally supersymmetric action for an $N=1$ chiral multiplet confined to a $4 \mathrm{~d}$ brane and coupled to $5 \mathrm{~d}$ supergravity in the bulk. We use the Noether method. That is, starting with a globally supersymmetric action for the brane multiplet we systematically add new terms to the action and supersymmetry transformations until the bulk+brane set-up becomes locally supersymmetric. We first work out all necessary zeroand two-fermion terms such that all two-fermion supersymmetric variations of the brane action cancel. The next step is to determine four-fermion terms from cancellation of four-fermion variations. In fact, the latter step will not be presented here, but see [11. It should be stressed however, that at the two-fermion level the brane action we construct is locally supersymmetric to arbitrary power in $1 / M_{5}$ expansion, where $M_{5}$ is the $5 \mathrm{~d}$ Planck scale. 
The 5 d bulk contains $N=2$ supergravity multiplet ${ }^{1}\left(e_{\alpha}^{a}, \psi_{\alpha}, \mathcal{A}_{\alpha}\right)$. For the flat (ungauged) 5d supergravity the action up to four-fermion terms reads 12

$$
\begin{gathered}
\mathcal{L}=M_{5}^{3} e_{5}\left[\frac{1}{2} R_{5}-i \overline{\psi_{\alpha}} \Gamma^{\alpha \beta \gamma} D_{\beta} \psi_{\gamma}-\frac{1}{4} \mathcal{F}_{\alpha \beta} \mathcal{F}^{\alpha \beta}+\frac{1}{6 \sqrt{6}} \epsilon^{\alpha \beta \gamma \delta \epsilon} \mathcal{A}_{\alpha} \mathcal{F}_{\beta \gamma} \mathcal{F}_{\delta \epsilon}\right. \\
\left.+\frac{3 i}{4 \sqrt{6}} \overline{\psi_{\alpha}} \Gamma^{\alpha \beta \gamma \delta} \psi_{\beta} \mathcal{F}_{\gamma \delta}+\frac{3 i}{2 \sqrt{6}} \overline{\psi_{\alpha}} \psi_{\beta} \mathcal{F}^{\alpha \beta}\right]
\end{gathered}
$$

while the supersymmetry transformations, up to three-fermion terms in $\delta \psi$ are given by

$$
\begin{aligned}
\delta e_{\alpha}^{a} & =\frac{i}{2} \overline{\psi_{\alpha}} \Gamma^{a} \epsilon+\text { h.c. } \\
\delta \psi_{\alpha} & =D_{\alpha} \epsilon-\frac{1}{4 \sqrt{6}}\left(\Gamma_{\alpha \beta \gamma}-4 g_{\alpha \beta} \Gamma_{\gamma}\right) \epsilon \mathcal{F}^{\beta \gamma} \\
\delta \mathcal{A}_{\alpha} & =-\frac{i \sqrt{6}}{4} \overline{\psi_{\alpha}} \epsilon+\text { h.c. }
\end{aligned}
$$

The fifth dimension is the orbifold $S_{1} / Z_{2}$ parametrized by $x_{5} \in[-\pi R, \pi R]$ with $Z_{2}$ acting as $x_{5} \rightarrow-x_{5}$. Under $Z_{2}$ the field components $e_{\mu}^{m}, e_{5}^{5}, \mathcal{A}_{5}$, $\psi_{\mu}^{+} \equiv P_{R} \psi_{\mu}$ and $\psi_{5}^{+} \equiv P_{L} \psi_{5}$ are even, $\psi\left(-x_{5}\right)=\psi\left(x_{5}\right)$, while $e_{\mu}^{5}, e_{5}^{m}, \mathcal{A}_{\mu}$, $\psi_{\mu}^{-} \equiv P_{L} \psi_{\mu}$ and $\psi_{5}^{-} \equiv P_{R} \psi_{5}$ are odd, $\psi\left(-x_{5}\right)=-\psi\left(x_{5}\right)$. At the orbifold fixed point $x_{5}=0$ we couple the $N=1$ chiral multiplet $\left[Q_{0}, \psi_{Q}\right]\left(P_{L} \psi_{Q}=\psi_{Q}\right)$. Of course the action for the matter $\left[Q_{\pi}, \psi_{Q_{\pi}}\right]$ at the orbifold fixed point $x_{5}=\pi R$ can be constructed analogously. The starting point for the Noether method is the action

$$
\mathcal{L}_{1}=e_{4} \delta\left(x_{5}\right)\left\{\partial_{\mu} Q_{0}^{\dagger} \partial^{\mu} Q_{0}+i \bar{\psi}_{Q} \gamma^{\mu} D_{\mu} \psi_{Q}\right\}
$$

invariant under global supersymmetry transformations

$$
\delta Q_{0}=\frac{1}{\sqrt{2}} \bar{\epsilon} \psi_{Q} \quad \delta \psi_{Q}=-\frac{1}{\sqrt{2}} i \gamma^{\mu} \partial_{\mu} Q_{0} \epsilon
$$

Under the transformations (44) but with $\epsilon$ depending on the 4 d coordinates $x_{\mu}$ the lagrangian of eq. (3) transforms as $\delta \mathcal{L}=\partial_{\mu} \epsilon j^{\mu}$, where $j^{\mu}$ is the Noether current of global supersymmetry (the supercurrent). In order to cancel this variation we need to couple one linear combination $\psi_{\mu}$ of the two bulk gravitinos $\psi_{\mu}^{+}(0), C{\overline{\psi_{\mu}^{-}}}^{-}(0)$ to the the supercurrent and identify the parameter $\epsilon$ with the corresponding combination of the two bulk supersymmetry transformation parameters, $\epsilon_{+}(0)$ and $C \bar{\epsilon}_{-}^{T}(0)$. In absence of brane sources for the gravitinos $\psi_{\mu}^{-}(0)=0$ and thus we choose $\psi_{\mu}=\psi_{\mu}^{+}(0)$. However, if such

\footnotetext{
${ }^{1}$ Our notation and conventions are summarized in Appendix A
} 
sources are present some other combination of the gravitinos couples to the brane matter. This is for example the case when gravitino brane mass terms are present, see the discussion in Appendix B.

Thus we add to the brane action the so-called Noether term,

$$
\mathcal{L}_{2}=-\frac{1}{\sqrt{2}} e_{4} \delta\left(x_{5}\right) \bar{\psi}_{Q} \gamma^{\mu} \gamma^{\rho} \partial_{\rho} Q_{0} \psi_{\mu}+\text { h.c. } .
$$

At the level of two-fermion terms there are still variations to be canceled. One originates from varying the gravitino in eq. (5),$\delta \psi_{\mu} \sim \mathcal{F}_{\mu 5}$, the other from variation of the vielbein in the kinetic terms of eq. (3). It turns out that the necessary modifications of the brane action can be concisely summarized as the redefinition of the graviphoton field strength. Namely, in the $5 \mathrm{~d}$ bulk action (11) and supersymmetry transformations (2) we replace $\mathcal{F}_{\mu 5}$ with $\hat{\mathcal{F}}_{\mu 5}$ defined as

$$
\begin{gathered}
\hat{\mathcal{F}}_{\mu 5}=\mathcal{F}_{\mu 5}+\frac{1}{M_{5}^{3}} \delta\left(x_{5}\right) j_{\mu}^{0}, \\
j_{\mu}^{0}=\frac{i}{\sqrt{6}}\left[Q_{0}^{\dagger} \partial_{\mu} Q_{0}-\partial_{\mu} Q_{0}^{\dagger} Q_{0}+\frac{i}{2} \bar{\psi}_{Q} \gamma_{\mu} \psi_{Q}\right],
\end{gathered}
$$

and modify the transformation law of the graviphoton by

$$
\delta \mathcal{A}_{5}=\frac{i}{\sqrt{12}} \delta\left(x_{5}\right) \bar{\psi}_{Q} \epsilon Q_{0}+\text { h.c. } .
$$

In other words we modify the Bianchi identity for the graviphoton field strength such that $\partial_{[\mu} \hat{\mathcal{F}}_{\nu] 5}=\frac{2 i}{\sqrt{6} M_{5}^{3}} \delta\left(x_{5}\right) \partial_{[\mu} Q_{0}^{\dagger} \partial_{\nu]} Q_{0}$. The replacement $\mathcal{F} \rightarrow \hat{\mathcal{F}}$ generates singular $\delta^{2}$ terms in the brane action. However such singular terms are absent in the low energy effective theory after integrating out the graviphoton $\mathcal{A}_{\mu}$. The reason for this is precisely the fact that the singular $\delta^{2}$ terms match the full square structure inside the graviphoton field strength. In the $5 \mathrm{~d}$ setup these singular terms provide for necessary counterterms to cancel divergences in certain one-loop diagrams [13]. No other singular terms arise in this construction. ${ }^{2}$

The on-shell action we derived by the Noether method differs from the brane action obtained in the off-shell formalism after eliminating the auxiliary fields [5]. In particular the kinetic terms of the gravity multiplet do not couple to the brane here. In Section 5 we will discuss this issue more carefully and argue that the two formalisms are related by a redefinition of the $5 \mathrm{~d}$ degrees

\footnotetext{
${ }^{2}$ The Noether construction of brane action in flat $5 \mathrm{~d}$ supergravity was also pursued in ref. [14 but their results differ from ours, notably by the absence of the full square structure.
} 
of freedom. Note that the choice of variables we use here is very convenient, as no singular $\delta^{n}$ terms are present in the on-shell action (except for the $\delta^{2}$ fitting the full square).

At this point all the two-fermion variations are canceled. More involved calculations are needed to work out four-fermion terms in the brane action as well as three-fermion modifications of the supersymmetry transformation laws. They are not necessary for the following analysis and will not be presented here. ${ }^{3}$ Indeed, we shall see that the form of the low energy $4 \mathrm{~d}$ supergravity (including one-loop corrections) can be read out from the terms we have already derived. Note also that once tree-level effective supergravity is known all the three- and four-fermion terms can be easily inferred by matching with the canonical $4 \mathrm{~d}$ supergravity lagrangian.

This construction of the brane world action can be carried over to the case of warped supergravity, that is, 5 d supergravity with a $U(1)$ subgroup of the $S U(2)$ R-symmetry group gauged by the graviphoton [15]. The 5d action can be obtained from the flat one in eq. (II) by replacing all the derivatives acting on the gravitino by

$$
D_{\alpha} \psi_{\beta} \rightarrow D_{\alpha} \psi_{\beta}+\frac{i}{2} k \epsilon\left(x_{5}\right) \Gamma_{\alpha} \psi_{\beta}+\frac{i \sqrt{6}}{2} k \epsilon\left(x_{5}\right) \mathcal{A}_{\alpha} \psi_{\beta}
$$

Analogous replacement should be done for $D_{\alpha} \epsilon$ in the gravitino transformation laws. Besides, the 5d bulk action (11) should be supplemented by

$$
\mathcal{L}=6 M_{5}^{3} k^{2} e_{5}
$$

Hence the gauging implies the presence of a negative cosmological constant in the bulk and so the gravitational background solution is $\mathrm{AdS}_{5}$. On the orbifold, the presence of the step function $\epsilon\left(x_{5}\right)$ induces additional variations proportional to the delta function,

$$
\delta \mathcal{L}=M_{5}^{3} e_{4} \delta\left(x_{5}\right) k\left[3 i \overline{\psi_{\mu}} \gamma^{\mu} \gamma^{5} \epsilon-\sqrt{6} i \overline{\psi_{\mu}} \gamma^{\mu \nu} \gamma^{5} \epsilon \mathcal{A}_{\nu}\right]\left(\delta\left(x_{5}\right)-\delta\left(x_{5}-\pi R\right)\right) .
$$

Canceling the first term requires the presence of the brane tension [16],

$$
\mathcal{L}=-6 M_{5}^{3} e_{5} k\left(\delta\left(x_{5}\right)-\delta\left(x_{5}-\pi R\right)\right) .
$$

and so the gravitational background in this set-up is precisely that of the Randall-Sundrum model [17]. In absence of brane matter the second term vanishes. When brane chiral multiplets are present the current $j_{\mu}^{0}$ in eq. (6)

\footnotetext{
${ }^{3}$ The complete action is given in ref. [11. See also this reference for coupling of $N=1$ gauge multiplets on the brane.
} 
acts as a source for the graviphoton so that it has a jump at the brane, $\mathcal{A}_{\mu} \sim \epsilon\left(x_{5}\right) \frac{1}{2 M_{5}^{3}} j_{\mu}^{0}$. Moreover, the Noether term (15) is a source for the negative parity gravitino. By equations of motion it behaves as $\gamma^{\mu \nu} \psi_{\nu}^{-} \sim$ $\frac{1}{2 \sqrt{2}} \epsilon\left(x_{5}\right) \gamma^{\rho} \gamma^{\mu} \psi_{Q} \partial_{\rho} Q_{0}$ near the brane. In the flat case $k \rightarrow 0$ these subtleties in boundary conditions do not affect the Noether construction at the level of two-fermion terms. But for $k \neq 0$ these boundaries conditions imply that the second term in eq. (10) is non-vanishing. ${ }^{4}$ It turns out that the necessary modification that cancels this term consists in multiplying the brane action by a $\left|Q_{0}\right|^{2}$ dependent factor,

$$
\omega_{0}\left(\left|Q_{0}\right|^{2}\right)=\frac{1}{1-\frac{k\left|Q_{0}\right|^{2}}{3 M_{5}^{3}}}
$$

The brane-world action up to four-fermion terms reads

$$
\begin{gathered}
\mathcal{L}=\mathcal{L}_{\text {bulk }}\left(\mathcal{F}_{\mu 5} \rightarrow \mathcal{F}_{\mu 5}+\frac{1}{M_{5}^{3}} \delta\left(x_{5}\right) j_{\mu}^{0}\right)+\delta\left(x_{5}\right) \mathcal{L}_{\text {brane }}, \\
j_{\mu}^{0}=\frac{i}{\sqrt{6}} \omega_{0}\left(Q_{0}^{\dagger} \partial_{\mu} Q_{0}-\partial_{\mu} Q_{0}^{\dagger} Q_{0}\right)-\frac{1}{2 \sqrt{6}} \omega_{0}^{2} \psi_{Q} \gamma_{\mu} \psi_{Q}, \\
\mathcal{L}_{\text {brane }}=-6 M_{5}^{3} k e_{5}+e_{4} \omega_{0}^{2}\left[\frac{1}{2} \partial_{\mu} Q_{0}^{\dagger} \partial^{\mu} Q_{0}\right. \\
\left.+\frac{i}{2} \bar{\psi}_{Q} \gamma^{\mu} D_{\mu} \psi_{Q}-\frac{1}{\sqrt{2}} e_{4} \delta\left(x_{5}\right) \bar{\psi}_{Q} \gamma^{\mu} \gamma^{\rho} \partial_{\rho} Q_{0} \psi_{\mu}+\text { h.c. }\right]
\end{gathered}
$$

One should also insert the factor $\omega_{0}$ into the transformation of the graviphoton in eq. (7). The action for the matter on the brane at $x_{5}=\pi R$ is analogous with $\delta\left(x_{5}\right) \rightarrow \delta\left(x_{5}-\pi R\right), k \rightarrow-k, Q_{0} \rightarrow Q_{\pi}, \psi_{Q} \rightarrow \psi_{Q_{\pi}}$.

One can further extend the model to include a non-trivial superpotential $W_{0}\left(Q_{0}\right)$ for the brane multiplet. The treatment of the boundary conditions is then much more involved and in this paper we only discuss some limiting cases. Howver this discussion is not necessary for our computation of oneloop corrections and so we shift it to Appendix B.

\section{$3 \quad$ Four-dimensional effective supergravity}

We move to discussing the form of the $4 \mathrm{~d}$ effective supergravity that describes the light degrees of freedom (those with masses below the compactification scale) of the $5 \mathrm{~d}$ theory compactified on the background

$$
d s^{2}=a^{2}\left(x_{5}\right) g_{\mu \nu} d x^{\mu} d x^{\nu}-\phi^{2} d x_{5}^{2} \quad a\left(x_{5}\right)=e^{-k \phi x_{5}} .
$$

\footnotetext{
${ }^{4} \mathrm{~A}$ similar treatment of the boundary conditions in supersymmetric variations was also employed in refs. [18, 19].
} 
The limit $k \rightarrow 0$ corresponds to flat compactification. The bosonic degrees of freedom are the $4 \mathrm{~d}$ metric $g_{\mu \nu}$, the radion $\phi \equiv \sqrt{g_{55}}$, the fifth component of the graviphoton $\mathcal{A}_{5}$ and the scalars on the two branes $Q_{0}$ and $Q_{\pi}$. The Kähler potential of the $4 \mathrm{~d}$ supergravity can be found by matching with the kinetic terms for those fields.

The kinetic terms for the metric component are obtained by inserting the background (14) into the 5d Einstein-Hilbert action,

$$
\mathcal{L}=\sqrt{-g} M_{p}^{2}\left[\frac{1}{2} \frac{1-a_{\pi}^{2}}{2 k \pi R} R(g)+\frac{3}{2} k \pi R a_{\pi}^{2}\left(\partial_{\mu} \phi\right)^{2}\right],
$$

where $a_{\pi}=e^{-k \pi R \phi}$ and $M_{p}^{2}=2 \pi R M_{5}^{3}$. To go the Einstein basis we need to perform the Weyl rescaling $g_{\mu \nu} \rightarrow \frac{1}{f_{E}(\phi)} g_{\mu \nu}^{(E)}$ with $f_{E}(\phi)=\frac{1-a_{\pi}^{2}}{2 k \pi R}$. Then the kinetic terms become

$$
\mathcal{L}=\sqrt{-g^{(E)}} M_{p}^{2}\left[\frac{1}{2} R(g)+\frac{3}{4 f_{E}(\phi)^{2}} a_{\pi}^{2}\left(\partial_{\mu} \phi\right)^{2}\right] .
$$

The kinetic terms in eq. (13) yield

$$
\mathcal{L}=\sqrt{-g^{(E)}} \frac{1}{f_{E}(\phi)}\left[\omega_{0}^{2} \partial_{\mu} Q_{0}^{\dagger} \partial^{\mu} Q_{0}+a_{\pi}^{2} \omega_{\pi}^{2} \partial_{\mu} Q_{\pi}^{\dagger} \partial^{\mu} Q_{\pi}\right]
$$

More care is needed to derive kinetic terms of $\mathcal{A}_{5}$. To do this consistently we need to integrate out the negative parity components of the graviphoton $\mathcal{A}_{\mu}$. The relevant part of the $5 \mathrm{~d}$ action is

$\mathcal{L}_{5 d}=\frac{1}{2 \phi} a^{2}\left(x_{5}\right) \sqrt{-g} M_{5}^{3}\left(\partial_{5} \mathcal{A}_{\mu}-\partial_{\mu} \mathcal{A}_{5}+\frac{1}{M_{5}^{3}} \delta\left(x_{5}\right) j_{\mu}^{0}+\frac{1}{M_{5}^{3}} \delta\left(x_{5}-\pi R\right) j_{\mu}^{\pi}\right)^{2}$.

The solution to the graviphoton equations of motion is $\mathcal{A}_{\mu}=\partial_{\mu} \mathcal{A}_{5} x_{5}+$ $\frac{1}{2 k} \epsilon\left(x_{5}\right) C_{\mu} a^{-2}\left(x_{5}\right)+\epsilon\left(x_{5}\right) D_{\mu}$. The boundary conditions $\mathcal{A}_{\mu}(0)=j_{\mu}^{0} / 2, \mathcal{A}_{\mu}(\pi R)=$ $-j_{\mu}^{\pi} / 2$ determine the integration constants and we find $C_{\mu}=-a_{\pi}^{2} \frac{\partial_{\mu} \mathcal{A}_{5}+\left(j_{\mu}^{0}+j_{\mu}^{\pi}\right) / M_{p}^{2}}{f_{E}(\phi)}$. Inserting this solution back into the $5 \mathrm{~d}$ action and integrating over $x_{5}$ yields

$$
\mathcal{L}_{4}=\frac{1}{2} M_{p}^{2} \sqrt{-g^{(E)}} \frac{a_{\pi}^{2}}{f_{E}(\phi)^{2}}\left(\partial_{\mu} \mathcal{A}_{5}+\frac{1}{M_{p}^{2}}\left(j_{\mu}^{0}+j_{\mu}^{\pi}\right)\right)^{2} .
$$

Note that the $\delta^{2}$ terms has canceled. The Kähler potential that reproduces the kinetic terms (16), (17) and (19) is given by $K=-3 \log \Omega$ where

$$
\Omega=\frac{1-e^{-k \pi R(T+\bar{T})}}{2 k \pi R}-\frac{1}{3 M_{p}^{2}}\left|Q_{0}\right|^{2}-\frac{1}{3 M_{p}^{2}} e^{-k \pi R(T+\bar{T})}\left|Q_{\pi}\right|^{2},
$$




$$
\begin{gathered}
\operatorname{Re} T=\phi-\frac{1}{2 k \pi R} \log \left(1-\frac{2 k \pi R}{3 M_{p}^{2}}\left|Q_{0}\right|^{2}\right)+\frac{1}{2 k \pi R} \log \left(1+\frac{2 k \pi R}{3 M_{p}^{2}}\left|Q_{\pi}\right|^{2}\right), \\
\operatorname{Im} T=i \sqrt{\frac{2}{3}} \mathcal{A}_{5} .
\end{gathered}
$$

One can check that also the remaining interaction terms in the brane-world action (13) fit the general structure of $4 \mathrm{~d}$ supergravity [20] with the Kähler potential of eq. (20). Generalization to an arbitrary number of brane matter multiplets with general kinetic terms is straightforward. It amounts to replacing $\left|Q_{i}\right|^{2}$ with arbitrary real functions $\Omega_{i}\left(Q_{i}^{n}\right)$

$$
\begin{gathered}
\Omega=\frac{1-e^{-k \pi R(T+\bar{T})}}{2 k \pi R}-\frac{1}{3 M_{p}^{2}} \Omega_{0}\left(Q_{0}^{n}\right)-\frac{1}{3 M_{p}^{2}} e^{-k \pi R(T+\bar{T})} \Omega_{\pi}\left(Q_{\pi}^{m}\right), \\
\operatorname{Re} T=\phi-\frac{1}{2 k \pi R} \log \left(1-\frac{2 k \pi R}{3 M_{p}^{2}} \Omega_{0}\left(Q_{0}^{n}\right)\right)+\frac{1}{2 k \pi R} \log \left(1+\frac{2 k \pi R}{3 M_{p}^{2}} \Omega_{\pi}\left(Q_{\pi}^{m}\right)\right) .
\end{gathered}
$$

In the flat limit $k \rightarrow 0$ we recover the well-known no-scale structure,

$$
\begin{gathered}
\Omega=\frac{T+\bar{T}}{2}-\frac{1}{3 M_{p}^{2}} \Omega_{0}\left(Q_{0}^{n}\right)-\frac{1}{3 M_{p}^{2}} \Omega_{\pi}\left(Q_{\pi}^{m}\right), \\
T=\phi+\frac{1}{3 M_{p}^{2}} \Omega_{0}\left(Q_{0}^{n}\right)+\frac{1}{3 M_{p}^{2}} \Omega_{\pi}\left(Q_{\pi}^{m}\right)+i \sqrt{\frac{2}{3}} \mathcal{A}_{5} .
\end{gathered}
$$

Furthermore, in the presence of brane superpotential $W_{0}\left(Q_{0}^{i}\right)$ and $W_{\pi}\left(Q_{\pi}^{i}\right)$ the superpotential of the effective $4 \mathrm{~d}$ supergravity reads

$$
W=W_{0}\left(Q_{0}^{i}\right)+e^{-3 k \pi R T} W_{\pi}\left(Q_{\pi}^{i}\right) .
$$

The Kähler potential derived here is the same function of $T$ and $Q$ as the one in ref. [6] (note that we use the definition of $\Omega$ that differs by a factor $-1 /\left(3 M_{p}^{2}\right)$ from that of ref [6]). However the definition of the modulus $T$ in terms of the $5 \mathrm{~d}$ degrees of freedom is different (in our formulation it is also a function the brane matter fields). Of course, at tree-level the physics (like moduli stabilization, transmission of supersymmetry breaking) is the same in both formalisms. In particular the Kähler potential in eq. (21) implies no tree-level mediation of supersymmetry breaking through the bulk (although for $k \neq 0$ it is not of the no-scale form). 


\section{One-loop corrections to the Kähler poten- tial}

We now use our on-shell formulation of the $5 \mathrm{~d}$ theory to compute one-loop corrections to the tree-level Kähler potential. From the point of view of the $4 \mathrm{~d}$ effective theory no symmetry protects the particular structure of $\Omega$ in eq. (21). We expect that $\Omega_{1 \text { loop }}=\Omega+\Delta \Omega$ and that $\Delta \Omega$ includes couplings other than those in eq. (21), for example higher powers of $e^{-k \pi R(T+\bar{T})}$ or contact terms between $Q_{0}$ and $Q_{\pi}$. These new terms will lead to mediation of supersymmetry breaking.

We first compute the one-loop effective action in the full $5 \mathrm{~d}$ theory and then match to $4 \mathrm{~d}$ effective supergravity with a Kähler potential $\Omega+\Delta \Omega$. The computation involves regularization of divergent expressions so we first discuss the most general structure of the counterterms in the Kähler poten-

tial. Since $\Omega=e^{-K / 3}$ is the coefficient of the Einstein-Hilbert term in the supergravity conformal frame,

$$
\mathcal{L}_{C}=\sqrt{-g^{C}} M_{p}^{2}\left[\frac{1}{2} \Omega R-3 \Omega_{\bar{m} n} \partial_{\mu} z_{\bar{m}}^{\dagger} \partial_{\mu} z_{n}-\frac{3}{4 \Omega}\left(\Omega_{\bar{m}} \partial_{\mu} z_{\bar{m}}^{\dagger}-\Omega_{n} \partial_{\mu} z_{n}\right)^{2}+\ldots\right]
$$

the possible counterterms are constrained by the most general form of the Einstein-Hilbert terms consistent with $5 \mathrm{~d}$ general coordinate invariance and locality,

$$
\mathcal{L}=C_{B} \sqrt{-g_{5}} R_{5}+C_{0}\left(Q_{0}\right) \delta\left(x_{5}\right) \sqrt{-g_{4}} R_{4}+C_{\pi}\left(Q_{\pi}\right) \delta\left(x_{5}-\pi R\right) \sqrt{-g_{4}} R_{4} .
$$

After compactification on the warped background eq. (14) and Weyl rescaling to the conformal frame $g_{\mu \nu} \rightarrow f_{C} g_{\mu \nu}^{C}$ this becomes

$$
\mathcal{L}=\left[C_{B} \frac{1-a_{\pi}^{2}}{2 k \pi R}+C_{0}\left(Q_{0}\right)+C_{\pi}\left(Q_{\pi}\right) a_{\pi}^{2}\right] f_{C} \sqrt{-g^{C}} R
$$

In our case $f_{C}=1-\frac{2 k \pi R}{3 M_{p}^{2}} \Omega_{0}\left(Q_{0}\right)$. Using eq. (21) we express $\phi$ in $a_{\pi}=e^{-k \pi R \phi}$ by $T$ and $Q$ and we obtain

$$
\begin{gathered}
\mathcal{L}=\left[\left(\frac{1}{2 k \pi R} C_{B}+C_{0}\left(Q_{0}\right)\right)\left(1-\frac{2 k \pi R}{3 M_{p}^{2}} \Omega_{0}\left(Q_{0}\right)\right)\right. \\
\left.+e^{-k \pi R(T+\bar{T})}\left(-\frac{1}{2 k \pi R} C_{B}+C_{\pi}\left(Q_{\pi}\right)\right)\left(1+\frac{2 k \pi R}{3 M_{p}^{2}} \Omega_{\pi}\left(Q_{\pi}\right)\right)\right] \sqrt{-g^{C}} R .
\end{gathered}
$$

We see that the coefficient of the Einstein-Hilbert term is of the same form as the Kähler potential (21). We are thus guaranteed that all divergences we 
encounter in the one-loop computation can be absorbed by renormalization of the parameters in the tree-level Kähler potential (21). In particular, these divergences are not relevant for the questions of supersymmetry breaking mediation through the bulk. On the other hand, any couplings in $\Delta \Omega$ that are different than those in the tree-level Kähler potential correspond necessarily to non-local operators in 5d theory and therefore they should be finite and UV insensitive.

Reference [6] derives a very useful expression for $\Delta \Omega$,

$$
\Delta \Omega \sim \int \frac{d^{4} k}{(2 \pi)^{4}} \sum_{n} \frac{1}{k^{2}} \log \left(k^{2}+m_{n}^{2}\right) .
$$

Before we compute $\Delta \Omega$ in our set-up we first present a simple derivation of eq. (28). In order to compute $\Delta \Omega$ it is sufficient to compute corrections to the Einstein-Hilbert term in the conformal frame and compare the result with eq. (24). Quite generally, a field of spin $j$ and mass $m$ contributes to one-loop renormalization of the Einstein-Hilbert term (in dimensional regularization):

$$
\Delta \mathcal{L}_{j}=n_{j} \frac{\Gamma(1-d / 2) m^{d-2}}{(4 \pi)^{d / 2}} \sqrt{-g} R,
$$

where $n_{0}=0$ for a conformally coupled scalar, $n_{1 / 2}=1 / 6$ for a Dirac fermion, $n_{1}=-1 / 3$ for a gauge boson, and $n_{3 / 2}+n_{2}=0$ for a summed contribution of a Dirac gravitino and a graviton. Specializing to the case of $5 \mathrm{~d}$ sugra, a $5 \mathrm{~d}$ hypermultiplet contains one Dirac fermion, a 5 d vector multiplet - one Dirac fermion and one gauge boson, while the gravity multiplet contains one gauge boson, one Dirac gravitino and one graviton at each KK level. Summing all these contributions we find:

$$
\Delta \Omega=\frac{1}{3}\left(-2-N_{V}+N_{H}\right) \frac{\Gamma(1-d / 2)}{M_{p}^{2}(4 \pi)^{d / 2}} \sum_{n} m_{n}^{d-2} .
$$

where $N_{V}$ and $N_{H}$ is the number of vector multiplets and hypermultiplets, respectively, and $m_{n}$ are the masses of the KK modes in the conformal frame as a function of background values of $T$ and $Q$. For $N_{V}=N_{H}=0$ this formula is equivalent to that in ref. [6] with the momentum integral evaluated using dimensional regularization.

We now apply the general formula (30) to the model considered in this paper. In the frame set by $g_{\mu \nu}$ in eq. (14) the KK spectrum is given by positive roots of the equation:

$$
J_{1}\left(\frac{m_{n}}{k}\right) Y_{1}\left(\frac{m_{n}}{k a_{\pi}}\right)-Y_{1}\left(\frac{m_{n}}{k}\right) J_{1}\left(\frac{m_{n}}{k a_{\pi}}\right)=0 .
$$


Note that in our set-up the KK spectrum $m_{n}$ is a function of the field $\phi$ only and is not modified by the presence of brane matter fields $Q$. Going to the conformal frame $m_{n} \rightarrow m_{n} f_{C}^{1 / 2}$, so $\Delta \Omega$ picks up an additional multiplicative factor $f_{C}^{d / 2-1}$.

Using the standard tools 21] we convert the sum over $\mathrm{KK}$ modes into a contour integral. The divergent part is of the form $f_{C}\left(C_{1}+a_{\pi}^{2} C_{2}\right)$ and can be absorbed into renormalization of the tree-level Kähler potential. The remaining finite part is given by

$$
\begin{gathered}
\Delta \Omega=\frac{4}{3 M_{p}^{2}(4 \pi)^{2}}\left(1-\frac{2 k \pi R}{3 M_{p}^{2}} \Omega_{0}\left(Q_{0}\right)\right) k^{2} a_{\pi}^{2} \int_{0}^{\infty} d y y \log \left(1-\frac{I_{1}\left(y a_{\pi}\right) K_{1}(y)}{K_{1}\left(y a_{\pi}\right) I_{1}(y)}\right) \\
a_{\pi}^{2}=e^{-k \pi R(T+\bar{T})} \frac{1+\frac{2 k \pi R}{3 M_{p}^{2}} \Omega_{\pi}\left(Q_{\pi}\right)}{1-\frac{2 k \pi R}{3 M_{p}^{2}} \Omega_{0}\left(Q_{0}\right)} .
\end{gathered}
$$

The Kähler potential $\Omega+\Delta \Omega$ contains all information about the contact terms between the hidden and observable sectors. In the limit of large warping, $a_{\pi} \rightarrow 0$ we can approximate $\Delta \Omega$ by:

$$
\Delta \Omega \approx-\frac{4 c k^{2}}{3 M_{p}^{2}(4 \pi)^{2}} e^{-2 k \pi R(T+\bar{T})} \frac{\left(1+\frac{2 k \pi R}{3 M_{p}^{2}} \Omega_{\pi}\left(Q_{\pi}\right)\right)^{2}}{1-\frac{2 k \pi R}{3 M_{p}^{2}} \Omega_{0}\left(Q_{0}\right)}+\mathcal{O}\left(a_{\pi}^{6} \log a_{\pi}\right),
$$

where $c=\int_{0}^{\infty} d y y^{3} \frac{K_{1}(y)}{2 I_{1}(y)} \approx 1.165$. On the other hand in the flat limit $k \rightarrow 0$ we find

$$
\Delta \Omega=-\frac{16 \zeta(3)}{3(4 \pi)^{2}} \frac{1}{\left(2 \pi R M_{p}\right)^{2}} \frac{1}{\left(T+\bar{T}-\frac{2}{3 M_{p}^{2}} \Omega_{0}\left(Q_{0}\right)-\frac{2}{3 M_{p}^{2}} \Omega_{\pi}\left(Q_{\pi}\right)\right)^{2}} .
$$

\section{On ambiguity in one-loop Kähler potential}

We now compare the result of our computation to the previous works on the subject [3, 5, 4]. For simplicity, we restrict to the flat limit $k \rightarrow 0$. Expanding the $\Delta \Omega$ in eq. (34) in powers of $\Omega_{i}$ we obtain

$$
\begin{gathered}
\Delta \Omega=-\frac{16 \zeta(3)}{3(4 \pi)^{2}} \frac{1}{\left(2 \pi R M_{p}\right)^{2}}\left[\frac{1}{(T+\bar{T})^{2}}+\frac{4}{3 M_{p}^{2}(T+\bar{T})^{3}}\left(\Omega_{0}\left(Q_{0}\right)+\Omega_{\pi}\left(Q_{\pi}\right)\right)\right. \\
\left.+\frac{4}{3 M_{p}^{4}(T+\bar{T})^{4}}\left(\Omega_{0}\left(Q_{0}\right)+\Omega_{\pi}\left(Q_{\pi}\right)\right)^{2}+\ldots\right] .
\end{gathered}
$$

The first term describes the Casimir energy [21], the second corresponds to radion mediation [3] and the last one to brane-to-brane mediation of supersymmetry breaking [5]. At this order, all the terms in eq. (35) are the same 
as those derived in the literature. However the full formula eq. (34) is clearly different than that in ref. [5] and the discrepancy enters at the cubic order in $\Omega_{i}$. As long as the brane sources are perturbative, the physical consequences of both formulations (summarized in eq. (35)) are the same. However when the brane sources are large (for $\Omega_{i}>2 \pi R \phi M_{5}^{3}$ ) conclusions derived in both formalisms may be completely different. In particular, from eq. (34) it is evident that a constant terms in the boundary Kähler potential, $\Omega_{i}=L+\ldots$, is equivalent to shifting $T$ by a constant and therefore has no physical significance. This is certainly different than in ref. [5] where a large value of $L$ was needed for obtaining positive soft mass terms.

The origin of this incompatibility can be traced to the different formulation of the $5 \mathrm{~d}$ brane-world theory. The technical issue that affects the one-loop computation is the fact that in the off-shell formulation $\Omega_{0}$ and $\Omega_{\pi}$ multiply brane kinetic terms of the gravity multiplet. These couplings remain after integrating out the auxiliary fields. On the other hand, in our purely on-shell Noether formulation such terms are absent. In order to understand this difference better, below we discuss supersymmetrization of a model with a brane Einstein-Hilbert term by means of the Noether procedure. For simplicity we restrict to the case where no brane matter is present. Thus we start with the brane lagrangian of the form

$$
\mathcal{L}=e_{4} M_{5}^{3} L \delta\left(x_{5}\right)\left[\frac{1}{2} R_{4}-i \bar{\psi}_{\mu}^{+} \gamma^{\mu \nu \rho} D_{\nu} \psi_{\rho}^{+}\right] .
$$

In ordinary $4 \mathrm{~d}$ supergravity this lagrangian would be supersymmetric up to four-fermion terms. But here $\delta \psi_{\mu} \sim \mathcal{F}_{\mu 5} \epsilon$ and so the variation of eq. (36) is non-zero already at the two-fermion level. To cancel it one has to add new zero- and two-fermion terms to eq. (36) as well as modify the supersymmetry transformation of the gravitino by terms proportional to $L \delta$. However once one arrives at a lagrangian in which all variations of order $L \delta$ cancel one finds that there are $L^{2} \delta^{2}$ variations that do not cancel. Therefore the Noether procedure must be continued and new singular terms of order $L^{2} \delta^{2}$ have to be added to eq. (36) to make the lagrangian supersymmetric at this order ${ }^{5}$. The story does not end at order $\delta^{2}$. In order to maintain supersymmetry singular terms with higher and higher powers of $\delta$ are needed. However one can notice that there is a certain pattern emerging. It turns out that all the terms obtained by the Noether procedure can be obtained from the bulk action

$$
\mathcal{L}=e_{5} M_{5}^{3}\left[\frac{1}{2} R_{5}-i \bar{\psi}_{\alpha} \Gamma^{\alpha \beta \gamma} D_{\beta} \psi_{\gamma}-\frac{1}{4} \mathcal{F}_{\alpha \beta} \mathcal{F}^{\alpha \beta}+\ldots\right]
$$

\footnotetext{
${ }^{5}$ In the following we ignore the mathematical subtleties involved in multiplication of distribution and manipulate $\delta$ 's as if they were ordinary c-numbers
} 
by a formal, singular redefinition of the $\phi \equiv e_{\dot{5}}^{5}$ component of the $5 \mathrm{~d}$ vielbein,

$$
\phi \rightarrow \phi+L \delta\left(x_{5}\right)
$$

In addition, one should assume that only positive parity fields multiplied by $\delta\left(x_{5}\right)$ survive in the brane action.

For example by Noether procedure we get a series of graviphoton brane kinetic terms

$$
\begin{gathered}
\mathcal{L}=\frac{1}{2 \phi} M_{5}^{3} e_{4}\left[\frac{L}{\phi} \delta\left(x_{5}\right)-\frac{L^{2}}{\phi^{2}} \delta\left(x_{5}\right)^{2}+\frac{L^{3}}{\phi^{3}} \delta\left(x_{5}\right)^{2}+\ldots\right] \mathcal{F}_{\mu 5}^{2} \\
=\frac{1}{2}\left(\frac{1}{\phi+L \delta\left(x_{5}\right)}-\frac{1}{\phi}\right) M_{5}^{3} e_{4} \mathcal{F}_{\mu 5}^{2}
\end{gathered}
$$

One can argue that the extremely singular term like the one in eq. (39) is indeed needed for supersymmetry. Indeed, the graviton and gravitino KK spectrum with brane kinetic terms of eq. (36) is given by solutions of

$$
\tan \left(\phi \pi R m_{n}\right)=-\frac{1}{2} m_{n} L
$$

For the graviphoton the equation of motion reads $\partial_{5}\left(\frac{\partial_{5} \mathcal{A}_{\mu}}{\phi+L \delta\left(x_{5}\right)}\right)+\phi m_{n}^{2} \mathcal{A}_{\mu}=0$ To cancel all $\delta$ 's we should arrange that $\partial_{5} \mathcal{A}_{\mu}$ behaves as $\sim \phi+L \delta\left(x_{5}\right)$ near the boundary. Matching this boundary condition with the bulk solution of the equations of motion we get precisely the quantization condition eq. (40). Getting this spectrum for the graviphoton would be impossible without brane $\mathcal{F}^{2}$ term or with any decent non-singular $\mathcal{F}^{2} \delta$ term. We conclude that for supersymmetrizing a $5 \mathrm{~d}$ model with a boundary Einstein-Hilbert term it is indeed necessary to include an infinite series of singular $\delta^{n}$ terms in the action.

We can now infer the relation between the brane-world action obtained by integrating out auxiliary fields in the off-shell formulation and the one obtained by our Noether procedure. The two are connected by a singular change of variables

$$
\phi \rightarrow \phi-\frac{1}{3 M_{5}^{3}} \Omega_{0}\left(Q_{0}\right) \delta\left(x_{5}\right)-\frac{1}{3 M_{5}^{3}} \Omega_{\pi}\left(Q_{\pi}\right) \delta\left(x_{5}-\pi R\right) .
$$

If the two formalisms are in fact equivalent up to a change of variables why the computation of loop corrections yields different results? The difference can be traced to ambiguity of defining the behavior of bulk fields near the $\delta$ sources. In the above example, after the redefinition (38) we kept only positive $Z_{2}$ parity fields in the brane action. But once we switch on a source of order $L \delta$, by equations of motions the negative $Z_{2}$ parity fields behave like $\sim$ 
$L \epsilon\left(x_{5}\right)$ near the boundary. We are then allowed to keep also boundary terms involving $Z_{2}$ odd fields, $\mathcal{L} \sim\left(\psi^{-}\right)^{2} \delta\left(x_{5}\right)$, provided we define the distribution $\delta\left(x_{5}\right) \epsilon^{2}\left(x_{5}\right)$ to be non-vanishing. Such terms affect the KK spectrum at the cubic order in $L$ and, by eq. (30), also the one-loop Kähler potential at higher order in $L$.

Concluding, the change of variable eq. (41) defines in fact a class of braneworld actions, depending on what regularization scheme we adopt to resolve the brane singularity. Physical predictions within this class of theories may differ at the third order in brane sources. As long as the brane sources are perturbative, the relevant physical quantities (e.g. soft mass terms) derived in both formulations are the same. However, if the brane sources are large (in the above example, if $L$ is bigger that the compactification length $2 \pi R \phi$ ) the low-energy observables may depend on how the brane singularity is regularized.

\section{Conclusions}

In this paper we used the Noether method to construct $5 \mathrm{~d}$ on-shell gauged supergravity coupled to chiral matter multiplets on the branes. This turned out to lead to a slightly different set-up than that derived from the more commonly used off-shell formulation. Certain singularities that appear after integrating out the auxiliary fields are absent in the purely on-shell Noether formulation. This is due to a different choice of the fundamental degrees of freedom in the $5 \mathrm{~d}$ theory.

Furthermore, we showed that our on-shell set-up allows for a simple computation of one-loop corrections. Comparison of our results with previous works showed an ambiguity in computation of the one-loop Kähler potential. This ambiguity is associated with arbitrariness in resolving the singularities associated with infinitely thin, delta-type branes. As long as the brane sources are small (the reference scale being $M_{5}^{3} 2 \pi R \phi$ ) this ambiguity has negligible effects on the low-energy physics. However in certain 5d models large brane sources are essential. One well-known example is the DvaliGabadadze-Porratti 22] model in which gravity is localized on a $4 \mathrm{~d}$ brane in a semi-infinite flat extra dimension. We conclude that there is a whole class of supersymmetric completions of the DGP model that yield different low-energy predictions. Another example are set-ups with a gravitino brane mass term, $\mathcal{L} \sim W \delta\left(x_{5}\right) \psi_{\mu}^{T} \gamma^{\mu \nu} \psi_{\nu}+$ h.c.. The limit $W \rightarrow \infty$ is sometimes considered as being equivalent to the set-up with supersymmetry broken by boundary conditions. In such limit there is also a continuous family of regularizations that results in different physics at low energies. 
We expect that the Noether method can be easily carried over to other brane-world models, for example to $6 \mathrm{~d}$ supergravity with matter on a brane of co-dimension two. This offers an opportunity to construct more general brane-world actions and study its low-energy phenomenology without a necessity of going through the off-shell calculus.

\section{Acknowledgments}

I would like thank Stefan Pokorski for numerous discussions and collaboration on some stages of this project. I am also grateful to Riccardo Rattazzi and Claudio Scrucca for useful discussions.

The author was partially supported by thr Polish KBN grant 2 P03B 129 24 for years 2003-2005 and by the EC Contract MRTN-CT-2004-503369 network "The Quest for Unification: Theory Confronts Experiment" (20042008). The stay at DESY is possible due to Research Fellowship granted by Alexander von Humboldt Foundation.

\section{Appendix A Notation and conventions}

We use the mostly minus metric signature $(+,-,-,-)$. The index conventions are the following: $5 \mathrm{~d}$ Einstein indices $\alpha, \beta, \gamma \ldots=0 \ldots 3,5,5 \mathrm{~d}$ Lorentz indices $a, b, c \ldots=0 \ldots 3,5,4 \mathrm{~d}$ Einstein indices $\mu, \nu, \rho \ldots=0 \ldots 3$, $4 \mathrm{~d}$ Lorentz indices $m, n, \ldots=0 \ldots 3$.

The $5 \mathrm{~d}$ vielbein is denoted by $e_{\alpha}^{a}$ and satisfies $e_{\alpha}^{a} e_{\beta}^{b} \eta_{a b}=g_{\alpha \beta} . e_{5}$ is the determinant of the $5 \mathrm{~d}$ vielbein, while by $e_{4}$ we denote the determinant of the $4 \mathrm{~d}$ vielbein induced at the boundary. Similarly, $R_{5}$ denotes the $5 \mathrm{~d}$ Ricci scalar, while $R_{4}$ denotes the Ricci scalar constructed from the 4 d vielbein induced at the boundary. The inverse vielbein $e_{a}^{\alpha}$ satisfies $e_{a}^{\alpha} e_{\beta}^{a}=\delta_{\alpha}^{\beta}$.

$5 \mathrm{~d}$ gamma matrices are denoted as $\Gamma^{a}$ while $4 \mathrm{~d}$ gamma matrices are denoted as $\gamma^{m}$. They satisfy $\left\{\Gamma^{a}, \Gamma^{b}\right\}=2 \eta^{a b}$ and $\left\{\gamma^{m}, \gamma^{n}\right\}=2 \eta^{m n}$. The connection between the two sets is given by $\Gamma^{m}=\gamma^{m}, \Gamma^{5}=i \gamma^{5}$. Furthermore $\Gamma^{\alpha}=e_{a}^{\alpha} \Gamma^{a}, \gamma^{\mu}=e_{m}^{\mu} \gamma^{m}$. The convention for $\gamma^{5}$ is $\gamma^{5}=\operatorname{diag}(-1,-1,1,1)$ and the chirality projection operators are $P_{L}=\left(1-\gamma^{5}\right) / 2, P_{R}=\left(1+\gamma^{5}\right) / 2$. All the fermions in $5 \mathrm{~d}$ and $4 \mathrm{~d}$ are in four-component Dirac notation (we don't use symplectic Majorana spinors). The $4 \mathrm{~d}$ charge conjugation matrix $C=i \gamma^{0} \gamma^{2} \gamma^{5}$ satisfies $C^{-1}=C^{T}=C^{\dagger}=-C, C \gamma^{m} C^{-1}=\left(\gamma^{m}\right)^{T}$. 


\section{Appendix B Brane action with a superpoten- tial}

One can extend the set-up studied in Section 2 to include a non-trivial superpotential $W_{0}\left(Q_{0}\right)$ for the brane multiplet. We start with the case of flat supergravity in the bulk. In addition to those of eq. (3), the terms present in the globally supersymmetric limit are the following

$$
\mathcal{L}_{3}=e_{4} \delta\left(x_{5}\right)\left[-\left|\frac{\partial W_{0}}{\partial Q_{0}}\right|^{2}+\frac{1}{2} \frac{\partial^{2} W_{0}}{\partial Q_{0}^{2}} \psi_{Q}^{T} C \psi_{Q}-\frac{1}{2} \frac{\partial^{2} \bar{W}_{0}}{\partial Q_{0}^{\dagger 2}} \bar{\psi}_{Q} C \bar{\psi}_{Q}^{T}\right]
$$

The supersymmetry transformation of the chiral fermion is supplemented

by $\delta \psi_{Q}=\frac{1}{\sqrt{2}} \frac{\partial \overline{W_{0}}}{\partial Q_{0}^{\dagger}} C \bar{\epsilon}^{T}$. In the presence of the superpotential the matter supercurrent is modified. The gravitino couples additionally as

$$
\mathcal{L}_{4}=-\frac{i}{\sqrt{2}} e_{4} \delta\left(x_{5}\right) \frac{\partial W_{0}}{\partial Q_{0}} \psi_{Q}^{T} C \gamma^{\mu} \psi_{\mu}+\text { h.c. . }
$$

Besides, up to four-fermion terms local supersymmetry requires one more term

$$
\mathcal{L}_{5}=e_{4} \delta\left(x_{5}\right)\left[-\frac{1}{2} W_{0} \bar{\psi}_{\mu} \gamma^{\mu \nu} C{\overline{\psi_{\nu}}}^{T}+\text { h.c. }\right]
$$

Furthermore one modifies the trasformation law of $\psi_{5}$ by

$$
\delta \psi_{5}=-\delta\left(x_{5}\right) W_{0} C \bar{\epsilon}^{T}
$$

The action on the other brane is analogous with $\delta\left(x_{5}\right) \rightarrow \delta\left(x_{5}-\pi R\right)$, $W_{0}\left(Q_{0}\right) \rightarrow W_{\pi}\left(Q_{\pi}\right)$. Again, no singular terms arise in this construction. One important comment is in order here. The gravitino brane mass term acts as a source for negative $Z_{2}$ parity gravitino so that $M_{5}^{3} \psi_{\mu}^{-} \sim \epsilon\left(x_{5}\right) W_{0} C{\overline{\psi_{\mu}^{+}}}^{T}$ near the boundary. Thus, in general, $\psi_{\mu}^{-}$can also couple to the brane matter. Therefore we have to reconsider the question which combination of the two bulk gravitinos should couple to the matter supercurrent in eq. (15) and eq. (B.2). It turns out that the answer depends on how the delta singularity is regularized. But whatever regularization we choose there is always one combination of the two bulk gravitinos $\epsilon\left(x_{5}\right) \psi_{\mu}^{+} \sin \theta+C{\overline{\psi_{\mu}^{-}}}^{T} \cos \theta$ that vanishes at the brane in the limit when the regulator is removed. Then the orthogonal combination $\psi_{\mu}=\psi_{\mu}^{+} \cos \theta-\epsilon\left(x_{5}\right) C{\overline{\psi_{\mu}^{-}}}^{T} \cos \theta$ couples to the brane matter. The angle of rotation is given by $\theta=W_{0} /\left(2 M_{5}^{3}\right)+\mathcal{O}\left(W_{0}^{3}\right)$, where the higher order terms in $W_{0}$ are regularization dependent. Coupling this combination $\psi_{\mu}$ to the matter supercurrent yields also correct (consistent with the general 
supergravity action [20]) couplings in the $4 \mathrm{~d}$ effective action, up to $\mathcal{O}\left(W_{0}^{3}\right)$ terms. But another conclusion from this discussion is that the low-energy $4 \mathrm{~d}$ supergravity is regularization independent only up to terms cubic in the brane superpotential $W_{0}$.

A careful treatment of the boundary conditions for gravitinos is even more important when warped supergravity $(k \neq 0)$ is present in the bulk. Ref. [18] discussed this problem for the case when brane matter is absent and $W_{0}$ is a constant. It was found that the brane action has to be modified already at the purely bosonic level. The brane tension term is given by:

$$
\mathcal{L}=-6 M_{5}^{3} k e_{4} \delta\left(x_{5}\right) \frac{1-\frac{W_{0}^{2}}{4 M_{5}^{6}}}{1+\frac{W_{0}^{2}}{4 M_{5}^{6}}} \approx-6 M_{5}^{3} k e_{4} \delta\left(x_{5}\right)\left(1-\frac{W_{0}^{2}}{2 M_{5}^{6}}\right)+\mathcal{O}\left(W_{0}^{4}\right)
$$

There is a similar term on the other brane with $k \rightarrow-k, W_{0} \rightarrow W_{\pi}$. Therefore, in the presence of gravitino brane mass terms the Randall-Sundrum tuning between the bulk cosmological constant and the brane tension is lost. The background solution is then of the Randall-Karch type [23] with $\mathrm{AdS}_{4}$ symmetry of the $4 \mathrm{~d}$ spacetime. Note that is consistent with what we obtain in the low energy $4 \mathrm{~d}$ supergravity description. With the Kähler potential eq. (21) and the superpotential eq. (23) we obtain the scalar potential

$V=\frac{6 k \pi R}{f_{E}(\phi)^{2} M_{p}^{2}}\left(\left|W_{0}\right|^{2}-e^{-4 k \pi R \phi}\left|W_{\pi}\right|^{2}\right)$ which is what follows from the warped compactification with the brane tension of eq. (B.5).

The case when warped supergravity, brane matter and brane superpotentials are present simultaneously is technically more involved and will be left for future studies.

\section{References}

[1] L. Randall and R. Sundrum, Nucl. Phys. B 557 (1999) 79 arXiv:hep-th/9810155.

[2] G. F. Giudice, M. A. Luty, H. Murayama and R. Rattazzi, JHEP 9812 (1998) 027 arXiv:hep-ph/9810442.

[3] T. Gherghetta and A. Riotto, Nucl. Phys. B 623 (2002) 97 arXiv:hep-th/0110022.

[4] I. L. Buchbinder, S. J. J. Gates, H. S. J. Goh, W. D. . Linch, M. A. Luty, S. P. Ng and J. Phillips, Phys. Rev. D 70 (2004) 025008 arXiv:hep-th/0305169. 
[5] R. Rattazzi, C. A. Scrucca and A. Strumia, Nucl. Phys. B 674 (2003) 171 arXiv:hep-th/0305184.

[6] T. Gregoire, R. Rattazzi, C. A. Scrucca, A. Strumia and E. Trincherini, arXiv:hep-th/0411216. C. A. Scrucca, arXiv:hep-th/0412237.

[7] Z. Lalak and R. Matyszkiewicz, Phys. Lett. B 583 (2004) 364 arXiv:hep-th/0310269|. N. Maru and N. Okada, Phys. Rev. D 70 (2004) 025002 |arXiv:hep-th/0312148|. H. Abe, K. Choi and I. W. Kim, JHEP 0409 (2004) 001 arXiv:hep-th/0405100. P. Brax and N. Chatillon, Phys. Lett. B 598 (2004) 99 arXiv:hep-th/0407025. F. P. Correia, M. G. Schmidt and Z. Tavartkiladze, arXiv:hep-th/0410281. G. von Gersdorff, L. Pilo, M. Quiros, A. Riotto and V. Sanz, arXiv:hep-th/0411133. H. Abe and K. Choi, JHEP 0412 (2004) 069 arXiv:hep-th/0412174 . F. P. Correia, M. G. Schmidt and Z. Tavartkiladze, arXiv:hep-th/0501209.

[8] M. Zucker, Phys. Rev. D 64 (2001) 024024 arXiv:hep-th/0009083. M. Zucker, Fortsch. Phys. 51, 899 (2003).

[9] T. Fujita, T. Kugo and K. Ohashi, Prog. Theor. Phys. 106 (2001) 671 arXiv:hep-th/0106051. T. Kugo and K. Ohashi, Prog. Theor. Phys. 108 (2002) 203 |arXiv:hep-th/0203276.

[10] P. Horava and E. Witten, Nucl. Phys. B 475 (1996) 94 arXiv:hep-th/9603142.

[11] A. Falkowski, M.Sc. Thesis (2000) http://info.fuw.edu.pl/ ^afalkows/Pub/publikacje.html

[12] A. H. Chamseddine and H. Nicolai, Phys. Lett. B 96 (1980) 89. M. Gunaydin, G. Sierra and P. K. Townsend, Nucl. Phys. B 242 (1984) 244.

[13] E. A. Mirabelli and M. E. Peskin, Phys. Rev. D 58 (1998) 065002 arXiv:hep-th/9712214.

[14] G. A. Diamandis, B. C. Georgalas, P. Kouroumalou and A. B. Lahanas, Phys. Lett. B 602 (2004) 112 arXiv:hep-th/0402228.

[15] M. Gunaydin, G. Sierra and P. K. Townsend, Nucl. Phys. B 253 (1985) 573. M. Gunaydin and M. Zagermann, Nucl. Phys. B 572 (2000) 131 arXiv:hep-th/9912027|. A. Ceresole and G. Dall'Agata, Nucl. Phys. B 585 (2000) 143 arXiv:hep-th/0004111|. E. Bergshoeff, S. Cucu, T. de Wit, J. Gheerardyn, S. Vandoren and A. Van Proeyen, Class. Quant. Grav. 21 (2004) 3015 arXiv:hep-th/0403045. 
[16] T. Gherghetta and A. Pomarol, Nucl. Phys. B 586 (2000) 141 arXiv:hep-ph/0003129. A. Falkowski, Z. Lalak and S. Pokorski, Phys. Lett. B 491, 172 (2000) arXiv:hep-th/0004093. A. Falkowski, Z. Lalak and S. Pokorski, Phys. Lett. B 509, 337 (2001) arXiv:hep-th/0009167.

[17] L. Randall and R. Sundrum, Phys. Rev. Lett. 83 (1999) 3370 arXiv:hep-ph/9905221.

[18] J. Bagger and D. V. Belyaev, Phys. Rev. D 67, 025004 (2003) arXiv:hep-th/0206024.

[19] J. A. Bagger and D. V. Belyaev, arXiv:hep-th/0406126.

[20] E. Cremmer, B. Julia, J. Scherk, S. Ferrara, L. Girardello and P. van Nieuwenhuizen, Nucl. Phys. B 147 (1979) 105.

[21] E. Ponton and E. Poppitz, JHEP $0106 \quad$ (2001) 019 arXiv:hep-ph/0105021.

[22] G. R. Dvali, G. Gabadadze and M. Porrati, Phys. Lett. B 485 (2000) 208 arXiv:hep-th/0005016.

[23] A. Karch and L. Randall, JHEP $0105 \quad$ (2001) 008 arXiv:hep-th/0011156. 